GHANA JOURNAL OF DEPARTMENT OF HEALTH, PHYSICAL EDUCATION AND RECREATION, SPORTS AND DANCE (GJOHPERSD)

\author{
Volume 11, Year 2018
}

A JOURNAL OF THE DEPARTMENT OF HEALTH, PHYSICAL EDUCATION AND RECREATION (HPER)

UNIVERSITY OF CAPE COAST GHANA, WEST AFRICA 
Sports and Dance (GJOHPERSD)

\title{
EFFECTS OF AEROBIC TRAINING ON \\ CARDIOVASCULAR PARAMETERS OF DIABETIC PATIENTS ATTENDING MURTALA MUHAMMED SPECIALIST HOSPITAL, KANO NIGERIA
}

\author{
Darma, M. H. \& Emiola, L.M. \\ Department of Physical and Health Education \\ Bayero University, Kano \\ musadarma@gmail.com Phone No.+2348030445351
}

\begin{abstract}
This study investigated the effects of aerobic training on cardiovascular parameters of diabetic patients attending Murtala Muhammed Specialist Hospital, Kano (MMSHK). The population of this study consisted of 522 diabetic patients attending MMSHK. A sample of 24 type 1 and 24 type 2 diabetic patients were purposively selected and randomly assigned into experimental $(n=12)$ and control $(n=12)$ for both types 1 and type 2 diabetic patients. However only 40 of the subjects completed the study 10 each for type 1 and 2 experimental and control groups. The participants in the experimental groups were engaged in run/walk training three times (on alternate days) weekly for 16 weeks. Both experimental and control groups continued their medications. The control groups were advised to continue with their normal sedentary daily life. The systolic BP, diastolic BP, resting HR and $\mathrm{Vo}_{2}$ max of all the subjects were measured before and after the 16 weeks training. Analysis of Covariance (ANCOVA) was used to test the stated hypotheses at 0.05 level of significance. The results revealed a significant reduction in the systolic blood pressure, diastolic blood pressure, resting HR, while the Vo2max increased significantly after the training among the experimental groups both type 1 and type 2 diabetic patients. The implication of the findings is that engaging diabetes patients both type 1 and type 2 in regular aerobic exercises will surely assist in the management and control
\end{abstract}


Darma, M. H, \& Emiola . L.M. of the illness due to the prevention of obesity which is known factor of diabetes. Based on the findings of the this study, it is recommended among others, that aerobic exercise (walk/run) should be used as an intervention in the management of both type 1 and 2 diabetes.

Key words: Cardiovascular parameters, Diabetic patients, Type 1 diabetes, Type 2 diabetes, Aerobic training. 
Effects of Aerobic Training on Cardiovascular Parameters of Diabetic Patents attending Murtala Muhammed specialist Hospital, Kano Nigeria

\section{Introduction}

Exercise has multiple short-term and long-term effects on the cardiovascular system. Cardiovascular system is also called the circulatory system. It is made up of the heart, arteries, veins and capillaries. The primary role of the cardiovascular system is to circulate oxygen-rich and glucose-rich blood to cells and to remove cellular waste products such as carbon dioxide from the muscles (Fujimoto, Prasad, \& Hastings 2010). Donges, Duffield, and Drinkwater, (2010) stated that the factors regulating cardiovascular function during exercise are complex and diverse.

At the initial stage of aerobic exercise, blood pressure increases as the cardiovascular system works to deliver more oxygen and glucose to working muscles. A long term adaptation to aerobic exercise is a de crease in both systolic and diastolic blood pressures during rest and during sub-maximal exercise. The American College of Sports Medicine encourages regular aerobic exercise as a method of controlling arid reducing high blood pressure (Waxman \& Nesto, 2002).

This study was therefore conducted to find out if aerobic training will have any effects on the cardiovascular parameters: Resting BP both systolic and diastolic BP, Resting HR and Max oxygen consumption (Vo2max), of insulin and non-insulin dependent diabetes patients attending Murtala Muhammad Specialist Hospital, Kano.

\section{Methods}

\section{Participants}

The participants for this study consisted of 48 diabetic patients, both insulin and non insulin dependent, drawn through purposive sampling technique. The selection was based on volunteers (those who were willing and consented to participate in the exercise training).

Twenty four (24) each were insulin dependent and non insulin dependent patients. Each group was then randomly assigned into the experimental and control groups. Thus the eventual four groups had 12 participants each.

Variables Measured 
The variables measured were: Blood pressure, systolic and diastolic BP, resting heart rate and Vo2max.

Training Procedure

There were four independent groups: Experimental and Control groups from insulin and non-insulin dependent diabetic patients. The control groups from both insulin and non-insulin dependent diabetic patients were not engaged in any form of formal physical training. They went about their regular day to day activities. The experimental groups of both insulin and non-insulin dependent diabetic patients went through 16 weeks of aerobic training (run/walk exercise) at Sani Abacha Stadium Kano, three alternate days a week at a progressive intensity of $50 \%$ to $80 \%$ of the maximal heart rate of the participants, as recommended by ISAK (2006). Adhama (2014), conducted a study on eight weeks using $45 \%$ - 85\% maximum heart rate of diabetics patients, while Yar'zenver (2008), also conducted a study for twelve weeks using 40\% - 80\% Maximal Heart Rate. However the Training Heart Rate of the current study was $50 \%$ to $80 \%$ of their Maximal Heart Rates . The training zone of a 37 years old thus as follows:

Maximal Heart Rate MHR $=220-37=183$ beats per minute Initial Training Heart Rate IHR $=183 \times .50=92$ beats per minute Final Training Heart Rate FHR $=183 \times .80=146$ beats per minute. Each of the participants wore a digital heart rate wrist watch. Each participant also had an orientation on his target heart rate based on his maximal heart rate. For the convenience of the participants, the training programme was conducted from 7am- 8am of every Monday, Wednesday and Friday. Before commencing the training, each participant was allowed 5-10 minutes warm-up (legs, hands and neck stretching and sit and reach exercises).

The percent heart rate range method was used and thus the three progressions (that is initial, improvement and maintenance) of each participant were individualized during the period of run/walk exercise. During the exercise with the help of four trained research assistants spread round the track, care was taken to ensure prompt identification of such signs and symptoms as weakness, dizziness and lack of muscular coordination. Participant with any of these 
Effects of Aerobic Training on Cardiovascular Parameters of Diabetic Patents attending Murtala Muhammed specialist Hospital, Kano Nigeria

signs was asked to slow down or stop for recovery before continuing. HR was taken prior to the start and at the end of the training to ensure the training intensity was adhered to. Intensity was progressively increased every 2 weeks by $5 \%$ from the $50 \%$ MHR till the $80 \%$ FHR was achieved.

The post-test measurements were taken at the end of the 16 weeks for both the experimental and the control groups. Two laboratory technologists from main lab of MMSHK severed as special research assistants who recorded the pre and post exercise cardiovascular parameters.

The Analysis of Covariance (ANCOVA) was used to analyse the data collected at 0.05 level of confidence.

\section{Results and Discussion}

The study was conducted with 48 diabetic participants, 10 from each of the four (4) groups, but in the course of the study 40 diabetic participants completed the study, and their scores (pre and post tests) were analyzed. 
The findings are presented as follows:

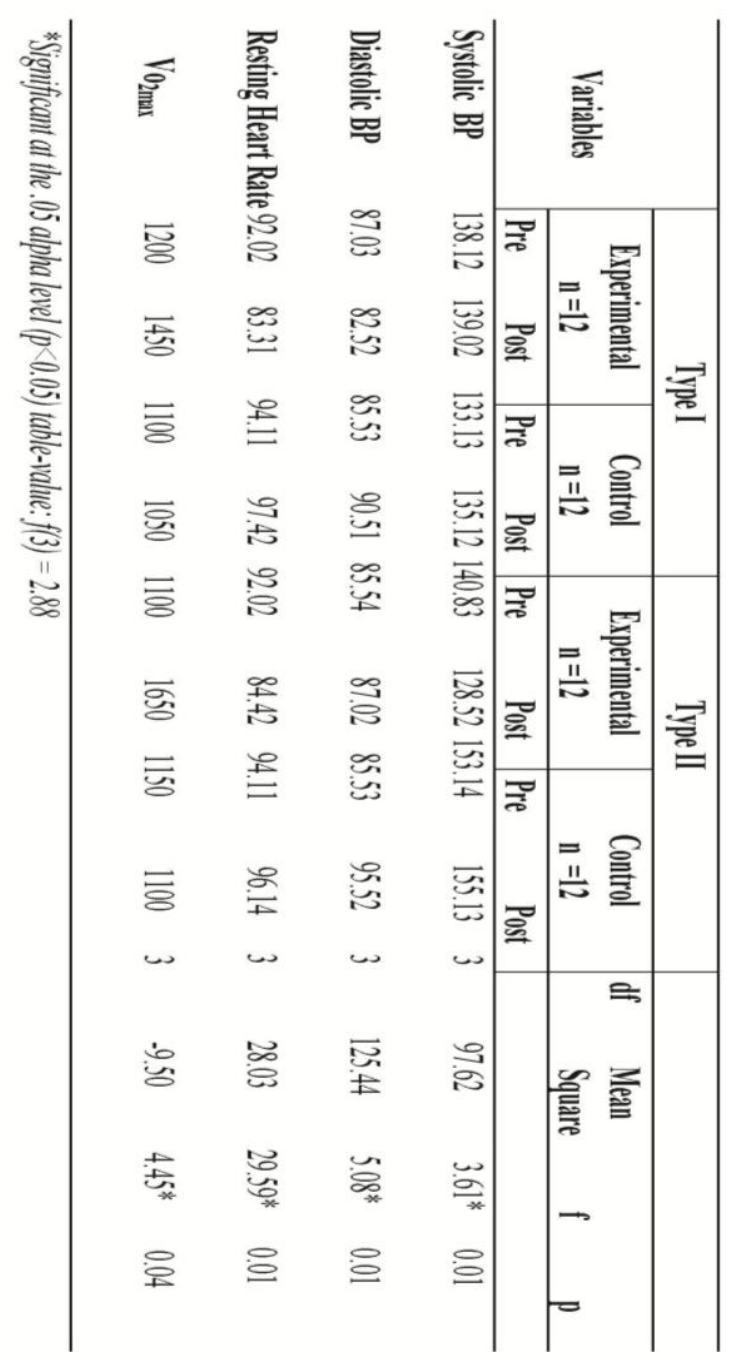

Table 1 indicates a significant $(\mathrm{P}<0.05)$ effect of aerobic training on all the selected cardiovascular parameters: The results showed significant reductions in the systolic $\mathrm{BP}$, diastolic $\mathrm{BP}$ and resting HR after the sixteen weeks of aerobic training, while the Vo2max of both the insulin and non-insulin dependent diabetic participants significantly increased after the 16 weeks of aerobic training. Due 
Effects of Aerobic Training on Cardiovascular Parameters of Diabetic Patents attending Murtala Muhammed specialist Hospital, Kano Nigeria

to significant effects of aerobic training on systolic BP, diastolic BP, HR and Vo2max of both the insulin and non-insulin dependent diabetic participants, the null hypothesis was rejected.

Table: 2: Summary of Post-Hoc Analysis on Cardiovascular Parameters among Groups

\begin{tabular}{|c|c|c|c|}
\hline Variables & & Groups & Mean difference \\
\hline & Prob. & & \\
\hline .Systolic BP & & Type I Experimental & 6.35 \\
\hline & $.030 *$ & & \\
\hline & & Type II Experimental & 8.04 \\
\hline & $.002 *$ & & \\
\hline & & Type II Control & .474 \\
\hline & & Type I Control & 3.21 \\
\hline .925 & & & \\
\hline Diastolic BP & & Type I Experimental & 5.88 \\
\hline & & Type II Experimental & 5.84 \\
\hline & & Type II Control & .217 \\
\hline .371 & & & \\
\hline & & Type I Control & .024 \\
\hline 635 & & & \\
\hline Resting HR & & Type I Experimental & 9.09 \\
\hline & $.001 *$ & & \\
\hline & $.002 *$ & Type II Experimental & 8.85 \\
\hline & & Type II Control & 129 \\
\hline & & Type I Control & 233 \\
\hline 441 & & & \\
\hline Vo2max & & Type I Experimental & 22.09 \\
\hline & $.001 *$ & & \\
\hline & $.007 *$ & Type II Experimental & 16.07 \\
\hline
\end{tabular}


Table 2 shows the summary of the post hoc analysis among the groups. The post hoc analysis showed that even though both the insulin dependent and the non-insulin dependent diabetes mellitus patients had significant reductions in the blood pressures and resting heart rate, after the training, the Type II group had more significant reduction than the type I participants in their systolic BP, while the type I participants had more significant reduction in their diastolic BP and the Resting HR respectively. On the other hand, the Vo2max of the type I group increased significantly more than that of the type II group.

\section{DISCUSSION}

The results of this study indicated a significant reduction of the systolic BP, of the experimental groups of insulin dependent and non-insulin dependent diabetics. This finding did agree with the works of Wokoma (2011), who in his study on hypertension in non-insulin dependent diabetics in Kano Nigerians, found that there were significant reductions in the systolic BP of the trained groups, while the control groups had a significant increase in their systolic BP. Yasuda, et al. (1992), in his study on effects of exercise on systolic and diastolic BP in middle-aged asymptomatic non-insulin-dependent diabetics, also found that there was a significant decrease in systolic BP of the exercise group and a significant increase in the control group.

The current study also revealed a significant reduction in the diastolic BP, of the experimental groups of insulin dependent and non-insulin dependent diabetic patients after the 16 weeks of the aerobic training. This finding strongly corroborates the work of Wokoma (2011), who again found significant reductions in the diastolic BP of the experimental group, while the control group 
Effects of Aerobic Training on Cardiovascular Parameters of Diabetic Patents attending Murtala Muhammed specialist Hospital, Kano Nigeria

had significant increase in their diastolic BP. Poirier, et al. (2001), also documented an inverse relationship between levels of exercise and diastolic pressure.

Aerobic training was also found to have significant effect on the resting $\mathrm{HR}$, of the experimental groups of insulin dependent and non-insulin dependent diabetes mellitus patients in the current study. This finding is in consonance with the work of Fujimoto, et al. (2010), and Rakowski et al. (2006), who equally found significant reductions in their trained group, while the control group had a significant increase in resting HR.

In the current study, the 16 weeks aerobic training result in a significant increase in the VO2max of the insulin dependent and non-insulin dependent diabetes mellitus participants. The findings of this study is in agreement with the study of Goodyear and Kahn (2009), who discovered that regular aerobic training leads to a significant increase in $\mathrm{VO} 2 \mathrm{max}$.

On the whole, the aerobic training in the current study was significantly beneficial to the cardiovascular fitness of both the insulin and non-insulin dependent diabetic patients.

\section{CONCLUSION}

From the findings of this study, the following conclusion was drawn:

Systolic blood pressure, diastolic blood pressure and resting heart rate of both the insulin and non-insulin dependent diabetic patients attending Murtala Muhammad Specialist Hospital, Kano significantly reduced after sixteen (16) weeks of aerobic exercise. While their Vo2max (their aerobic capacity) significantly increased.

\section{Recommendation}

Based on the findings of this study it is recommended that regular aerobic exercises be included in the management of diabetes 


\section{References}

Adhama, A.I. (2014). Cardiovascular Adaptation of Diabetic and Non Diabetic Stroke Patients to 8 weeks of Aerobic Exercise in selected Hospitals in Kano Metropolis. Unpublished Msc Dissertation Department of Physical and Health Education Bayero University, Kano

Donges, P. Duffield, S. \& Drinkwater, T. (2010). Physical Activity, Nutrition, and Chronic Disease. Medicine \& Science in Sports \& Exercise, 28, 335-349.

Fujimoto, N., Prasad, A., and Hastings, V. (2010). Cardiovascular effects of 1 year of progressive and vigorous exercise training in previously sedentary diabetes individuals older than 65 years of age. Circulation.122, 1797-1805.

Goodyear, L.J., and Kahn, B.B. (2009). Exercise, glucose transport, and insulin sensitivity and dietary control. Annu Rev Med. 49, 2.35-61.

International Society for the Advancement of Kinanthropometry (ISAK) (2006). International standards for anthropometric assessment. Accessed on 27th September, $2013 @$ http://isakonline.com/.

Poirier, P., Bogaty, P., Garneau, C., Marois, L., \& Dumesnil, J.G. (2001). Diastolic dysfnction in normotensive men with well-controlled type-2 diabetes: importance of maneuvers in echocardiographic screening for preclinical diabetic cardiomyopathy. Diabetes Care 24 $1,5-10$.

Waxman, S., and R. Nesto (2002). Cardiovascular Complications. In: N.B. Ruderman, J.T. Devlin, S.H. Schneider, and A. Kriska (eds.) Handbook of Exercise in Diabetes. Alexandria, VA: American Diabetes Association, 415431.

Wokoma, F.S. (2011). Hypertension in non-insulin dependent diabetic, Kano Nigerians. A comparative study of normotensive and hypertensive subgroups. Diabetes Kano Nigeria International 3, 57-58.

Yar'zenver, I.S. (2008). Effects of 8 week circle Ergometer exercise training on blood sugar and cholesterol in male 
Effects of Aerobic Training on Cardiovascular Parameters of Diabetic Patents attending Murtala Muhammed specialist Hospital, Kano Nigeria diabetic patients in Kano Metropolis, Nigeria. Unpublished Msc Dissertation Department of Physical and Health Education Bayero University, Kano. 